\title{
Deuxième journée dédiée aux travaux scientifiques des jeunes médecins de Guyane (JDIG) : Nos internes ont du talent Université de Guyane, Cayenne, Guyane
}

\author{
Second Day Dedicated to the Scientific Works of Young Doctors in French Guiana: \\ Our Residents' Got Talent \\ Université de la Guyane, Cayenne, French Guiana
}

\section{Epelboin $\cdot$ M. Douine $\cdot$ F. Henaff $\cdot$ R. Mutricy $\cdot$ A. Lucarelli $\cdot$ T. Bonifay $\cdot$ F. Niemetzky*}

Reçu le 14 février 2019; accepté le 15 février 2019

(C) Société de pathologie exotique et Lavoisier SAS 2019

\section{Éditorial}

\section{Epelboin ${ }^{1,2}$}

1. Unité des maladies infectieuses et tropicales, CHAR, Cayenne, Guyane française, France ; 2. Équipe EA 3593, écosystèmes amazoniens et pathologie tropicale, université de Guyane, Cayenne, Guyane française, France

Le 7 décembre 2018, a eu lieu sur le campus de l'université de Guyane, à Cayenne, la deuxième journée consacrée aux travaux scientifiques des jeunes médecins de Guyane. Cette session a rencontré un succès encore plus grand que la première édition, puisque plus de 150 personnes ont assisté à cette journée exceptionnelle. Exceptionnelle, car

\section{Epelboin $(\bowtie)$}

Unité des maladies infectieuses et tropicales, centre hospitalier Andrée-Rosemon (CHAR), Cayenne, F-97300 Guyane française, France epelboincrh@hotmail.fr

L. Epelboin · M. Douine

Équipe EA 3593, écosystèmes amazoniens et pathologie tropicale, université de Guyane,

Cayenne, Guyane française, France

\section{Douine}

Inserm CIC 1424, centre d'investigation clinique

Antilles-Guyane, CHAR,

F-97306 Cayenne, Guyane française, France

\section{F. Henaff}

Service de pédiatrie, CHAR,

Cayenne, Guyane française, France

F. Henaff · R. Mutricy

Service d'accueil des urgences,

CHAR, Cayenne, Guyane française, France elle a été l'occasion pour plus de 20 jeunes médecins de présenter leurs travaux de thèse ou de mémoire, en communication orale ou affichée, sur de multiples sujets pour la plupart enjeux de santé publique pour la Guyane. Les débats ont été riches, autour de sujets aussi variés que le paludisme, la leptospirose, les encéphalites, les morts inattendues du nourrisson, les causes de mort foetales in utero, les intoxications aiguës en pédiatrie, la prise en charge des infections urinaires en ville, l'épidémiologie des infarctus du myocarde, le parcours de soins des orpailleurs illégaux à Maripasoula, le saturnisme pédiatrique à Camopi et Trois-Sauts, parmi tant d'autres. Un public varié a assisté à cette journée scientifique, constitué de professionnels de santé hospitaliers ou de ville, des infirmières, des associatifs et même d'une

\footnotetext{
A. Lucarelli

Hôpital de jour adultes, CHAR,

Cayenne, Guyane française, France

T. Bonifay

Unité de consultation et de soins ambulatoires,

CHAR, Cayenne, Guyane française, France

T. Bonifay $\cdot$ F. Niemetzky

Département universitaire de médecine générale,

faculté de médecine Hyacinthe-Bastaraud,

université des Antilles, F-97100 Point-à-Pitre, Guadeloupe

F. Niemetzky

Pôle des centres délocalisés de prévention et de soins,

CHAR, Cayenne, F-97300 Guyane française, France

* Et les collaborateurs : Anna Auguste, Stéphanie Bernard, Olivier Bouchaud, Frédéric Bouteille, Paul Brousse, Gabriel Carles, Éric Caumes, Sophie Daltroff, Félix Djossou, Maryvonne Dueymes, Mélanie Gaillet, Basma Guarmit, Élise Martin, Christian Marty, Émilie Mosnier, Mathieu Nacher, Guillaume Odonne, Rémy Pignoux, Dominique Rousset, Alexandra Val, Guillaume Velut, Gaëlle Walter.
} 
vingtaine de collégiens. Plusieurs personnalités de Guyane et de métropole sont venues contribuer à valoriser ces journées : M. Cartiaux, directeur général de l'ARS Guyane, et M. Primerose, président de l'université de Guyane, sont venus introduire les journées, suivis des Prs Dueymes, Djossou et Nacher du centre hospitalier de Cayenne, ainsi que de nombreux modérateurs de différentes institutions de Guyane : institut Pasteur, CNRS, centre médical interarmées, réseau Périnat, préfecture de Guyane, infectiologues de l'Assistance publique des Hôpitaux de Paris sans compter Mme Alexandra Val, directrice de l'offre de soins de l'ARS et marraine de ces journées.

À noter que sur les 19 communications orales ou affichées présentées lors de la session de 2017, dix ont fait l'objet par la suite de publications dans des journaux anglophones et une dans un journal francophone, soit $58 \%$ des présentations. Un bémol au tableau cependant : deux de ces travaux ont été publiés à l'insu des thésards et en évinçant leur nom de la liste des auteurs, pratique d'un autre âge que l'on peine à comprendre. Espérons que les études présentées à la session 2018 fassent à leur tour l'objet de publications. Espérons également que cet événement à l'importance croissante se poursuive les années à venir et, qui sait, fasse des émules dans d'autres régions de France, de Navarre et des autres territoires ultramarins.

\section{Épidémiologie des parasitoses intestinales en Guyane française de 2011 à 2016}

\author{
A. Aboikoni ${ }^{1}$, M. Allaire ${ }^{1,2}$, D. Louvel ${ }^{3}$, D. Blanchet ${ }^{4}$, \\ T. Dao ${ }^{1}$, J.-F. Carod ${ }^{5}$, M. Demar ${ }^{4}$ \\ 1. Service d'hépatogastroentérologie, CHU Côte-de-Nacre, \\ Caen, France ; 2. UMR 1149, CRI, Paris, France ; 3. Service \\ de médecine B, CHAR, F-97300 Cayenne, Guyane fran- \\ çaise, France ; 4. Service de parasitologie et mycologie, \\ CHAR, F-97300 Cayenne, Guyane française, France ; 5. Ser- \\ vice de biologie, centre hospitalier de l'Ouest guyanais, \\ Saint-Laurent-du-Maroni, F-97320 Guyane française, \\ France \\ e-mail : a.aboikoni@gmail.com
}

La Guyane française, de par son climat équatorial et ses habitudes socioculturelles, est exposée aux parasitoses intestinales (PI). Une seule étude épidémiologique ciblée sur les populations amérindiennes a été publiée à l'heure actuelle. L'objectif de cette étude était de caractériser les PI dans ce département d'outre-mer afin d'aider à la mise en place de stratégies préventives.

L'étude est fondée sur le recueil rétrospectif de tous les prélèvements parasitologiques analysés entre 2011 et 2016 dans les laboratoires des deux principaux centres hospitaliers de Guyane réalisant des études parasitologiques. Les don- nées disponibles dans les registres étaient l'âge, le sexe, le lieu de résidence, la date et le type de prélèvement réalisé. Le territoire était divisé en régions en prenant en compte les habitudes socioculturelles des habitants. L'Est guyanais comprenait les villes de Camopi, d'Ouanary et de SaintGeorges ; l'Agglomération du centre littoral celles de Cacao, de Cayenne, de Kaw, de Kourou, de Macouria, de Matoury, de Montsinery, de Régina, de Rémire-Montjoly, de Roura ; le Littoral celles d'Iracoubo et de Sinnamary ; l'Ouest guyanais celles d'Apatou, d'Awala-Yalimapo, de Grand-Santi, de Mana, de Maripasoula, de Papaïchton, de Saint-Jean et de Saint-Laurent-du-Maroni et la région du Centre les villes de Saint-Élie et de Saul.

La saison des pluies était définie de décembre à juin. L'indice parasitaire (IP) correspondait au nombre de patients parasités sur le nombre total de patients étudiés. Un même patient n'était considéré qu'une seule fois pour le calcul de l'IP, quel que soit le nombre de prélèvements.

Quinze mille deux cent vingt prélèvements de 9555 patients différents ont été étudiés, 2877 d'entre eux étaient positifs chez 1482 patients (2 724 examens parasitologiques des selles et 153 liquides digestifs positifs). L'IP moyen était de $15,5 \%$ sans variation significative entre les années 2011 (18,4\%) et 2016 (17,3\%). Les patients parasités étaient majoritairement des hommes (66,7\%), âgés de 18 à 65 ans (69,2\%), de l'Agglomération du centre littoral $(49,4 \%)$ et de l'Ouest guyanais $(37,4 \%)$, diagnostiqués à la saison des pluies $(60,3 \%)$. Les trois parasites pathogènes les plus fréquents étaient Ancylostoma sp. $(25,7 \%)$, Strongyloides stercoralis $(11,1 \%)$ et Giardia intestinalis (11,0\%). Ancylostoma sp. était le plus fréquent dans toutes les zones géographiques, excepté sur les petites communes créoles du littoral, sans différence entre zones urbaine et rurale malgré le mode de transmission transcutanée, probablement en relation avec le fort contingent en zone urbaine d'immigrés des pays frontaliers et des mouvements de populations des zones rurales vers les grandes villes. Giardia intestinalis était plus fréquent dans l'Est et l'Ouest du territoire, à proximité des fleuves probablement du fait de l'ingestion d'eau contaminée, à la saison des pluies, chez les enfants de moins de cinq ans. 24,3\% des patients étaient polyparasités, principalement dans l'Est guyanais où il existe une forte population amérindienne répartie dans de petits villages enclavés dans la forêt amazonienne et difficilement accessibles.

L'IP moyen de $15 \%$ était plus élevé que celui des Antilles françaises. La sous-estimation liée au recueil des cas symptomatiques et l'absence de diminution de l'indice entre 2011 et 2016 témoignent de l'importance de ce problème de santé publique en Guyane qui justifierait la mise en place d'un plan de lutte global contre les PI qui apparaît impérative. 


\section{Parcours de soins des orpailleurs illégaux de Guyane française : du premier symptôme à la consultation médicale au centre de santé de Maripasoula}

\author{
A.-L. Aucouturier ${ }^{1}$, G. Walter ${ }^{2}$, G. Odonne ${ }^{3}$, M. Douine ${ }^{4}$ \\ 1. Pôle des centres de prévention et de soins, CHAR, \\ Cayenne 97306, Guyane française ; 2. Unité des maladies \\ infectieuses et tropicales, CHAR, Cayenne 97306, Guyane \\ française ; 3. LEEISA (Laboratoire écologie, évolution, \\ interactions des systèmes amazoniens), CNRS, Université \\ de Guyane, IFREMER, 275 route de Montabo, 97300, \\ Cayenne ; 4. Centre d'investigation clinique Antilles \\ Guyane, INSERM CIC 1424, CHAR, Cayenne 97306, \\ Guyane française \\ e-mail : aucouturier.annelaure@gmail.com
}

En Guyane française, la population des orpailleurs illégaux est peu étudiée sur le plan sanitaire. Des études préalables montrent pourtant qu'elle est sujette à des pathologies spécifiques comme le paludisme, le béribéri ou la leptospirose. Mieux connaître les pathologies et les parcours de soins de ces personnes permettrait aux professionnels de santé en contact avec elles d'adapter leur prise en charge.

Une étude prospective, descriptive et monocentrique a été menée au dispensaire de Maripasoula, commune située sur le fleuve Maroni qui sépare la Guyane du Suriname, à l'aide d'un questionnaire sur les caractéristiques sociodémographiques, l'activité et le parcours de soins de cette population. Des données médicales complémentaires ont été recueillies auprès des médecins.

D'août à novembre 2016, 63 personnes ont été incluses dans l'étude, avec un sex-ratio $\mathrm{H} / \mathrm{F}$ de 1,25 et une moyenne d'âge de 42 ans, originaires majoritairement du Brésil $(61 / 63)$ et parlant portugais couramment (62/63). La plupart des patients inclus avaient déjà consulté dans ce dispensaire (49/63) - dont ils étaient distants de 5 à 72 heures de trajet sans pour autant avoir de couverture sociale française (62/63). La majorité d'entre eux avait eu recours à l'automédication (55/63) avant la consultation, dont surtout des antalgiques (23/63), des anti-inflammatoires et corticoïdes $(16 / 63)$, des anti-infectieux (15/63) et des plantes médicinales (13/63). Ils déclaraient un retard d'accès aux soins $(51 / 63)$, souvent confirmé et estimé de modéré à important par le médecin (28/63).

Ces résultats indiquent qu'il existe un défaut d'accès aux soins des travailleurs de l'orpaillage illégal de Guyane avec un retard de prise en charge. Ils ont souvent recours à l'automédication avec un risque d'inefficacité, d'interactions médicamenteuses et d'émergence de résistances aux antimicrobiens.

\section{État des lieux des rapports sexuels chez les adolescents de la population multiethnique en Guyane : facteurs associés aux rapports précoces}

G. Ayhan ${ }^{1}$, L. Martin ${ }^{1}$, M. Levy-Loeb ${ }^{1}$, S. Thomas ${ }^{1}$, G. Euzet ${ }^{2}$, A. Van Melle ${ }^{1}$, M.-C. Parriault ${ }^{1}$, C. Basurko ${ }^{1}$, M. Nacher ${ }^{1,3}$

1. Centre d'investigation clinique Antilles Guyane, INSERM CIC 1424, CHAR, Cayenne 97306, Guyane française ; 2. Service de santé scolaire, Rectorat de la Guyane, Cayenne, Guyane française ; 3. COREVIH Guyane, CHAR, Cayenne 97306, Guyane française

e-mail : guelen.ayhan@gmail.com

La Guyane est considérée comme une zone d'épidémie généralisée pour l'infection par le VIH. Ce territoire est composé d'une population très jeune avec environ $45 \%$ des habitants âgés de moins de 20 ans. Retarder l'âge du premier rapport sexuel est un objectif majeur selon Onusida de la lutte contre l'infection par le VIH chez les adolescents. L'objectif de cette étude était de décrire l'âge au premier rapport sexuel et les facteurs associés à sa précocité chez les adolescents scolarisés en Guyane.

Une enquête de connaissances, attitudes et pratiques visà-vis de l'infection par le VIH a été menée en 2011 et 2012, à l'aide d'un autoquestionnaire, parmi les adolescents de 13 à 17 ans vivant sur le littoral et le fleuve Maroni. Un rapport sexuel précoce était défini comme survenant avant l'âge de 16 ans révolus.

Au total, 1603 étudiants ont participé à cette étude. L'âge médian du premier rapport était de 12,1 ans pour les garçons et de 13,9 ans pour les filles parmi les $60 \%$ d'adolescents déclarant avoir déjà eu un rapport sexuel. Parmi ceux-ci, $90 \%$ déclaraient avoir eu un rapport sexuel précoce. Les facteurs de risque associés au rapport sexuel précoce chez les hommes étaient : vivre sur le fleuve Maroni, déclarer une autre langue que le français comme langue maternelle, ne pas pratiquer de religion, consommer de l'alcool, consommer du cannabis et avoir une mauvaise attitude visà-vis de l'utilisation du préservatif. Pour les filles, les facteurs associés aux rapports sexuels précoces étaient un premier partenaire sexuel plus âgé, avoir eu plus de trois partenaires sexuels et la rupture du préservatif.

La mise en œuvre de politiques de santé publique en adéquation avec les spécificités sociodémographiques objectivées localement est nécessaire pour sensibiliser et améliorer l'appréciation des adolescents face aux risques liés à l'activité sexuelle précoce et aux maladies sexuellement transmissibles. 


\section{Risque suicidaire chez les prisonniers en Guyane : prévalence et facteurs de risque}

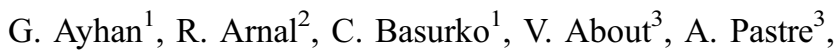
E. Pinganaud ${ }^{2}$, D. Sins ${ }^{2}$, L. Jehel ${ }^{4}$, B. Falissard ${ }^{5}$, M. Nacher ${ }^{1,6}$

1. Centre d'investigation clinique Antilles Guyane, INSERM CIC 1424, CHAR, Cayenne 97306, Guyane française ; 2. Unité fonctionnelle de psychiatrie intra-carcérale, CHAR, Cayenne 97306, Guyane française ; 3. Unité de soins et de consultations ambulatoires, CHAR, Cayenne 97306, Guyane française ; 4. Service de psychiatrie, CHU de Martinique, Fort-de-France, Martinique, France ; 5. Équipe IPSOM, INSERM 1178, Paris, France ; 6EA3593, Université de Guyane, Cayenne, Guyane française, France

e-mail : guelen.ayhan@gmail.com

Jusqu'à aujourd'hui, peu d'études ont analysé le risque suicidaire en prison à la fois dans les pays développés et encore moins dans les pays en voie de développement. Et pourtant, le taux de suicide en prison a augmenté, et les mécanismes restent inconnus. L'objectif principal était d'évaluer la prévalence des pathologies psychiatriques chez les prisonniers du centre pénitentiaire de Rémire-Montjoly, la seule prison de Guyane. L'objectif secondaire était d'estimer le risque suicidaire et ses facteurs prédictifs chez les détenus.

Afin d'évaluer le risque suicidaire dans la prison en Guyane, nous avons inclus tous les nouveaux arrivants éligibles dans notre étude entre septembre 2013 et décembre 2014. Les critères d'inclusion étaient : être majeur, ne pas être sous tutelle ou curatelle et avoir donné un avis de non-opposition. Nous avons utilisé le Mini International Neuropsychiatric Interview (MINI) afin de repérer des troubles psychiatriques et recueillir des données sociodémographiques. Il s'agit d'un test psychométrique structuré, développé afin de pouvoir diagnostiquer 17 troubles psychiatriques selon les critères du Manuel de diagnostic et de statistique (DSM)-V. Sa validité et sa fiabilité ont été confirmées par plusieurs études.

$\mathrm{Au}$ total, 707 prisonniers ont été inclus dans notre étude. Nous avons mis en évidence que le risque suicidaire chez les détenus (13,2\%) était moins élevé en Guyane qu'en France métropolitaine (40,3\%) ou en Martinique (26\%). Les facteurs de risque identifiés sont les troubles psychiatriques dont la dépression $(\mathrm{OR}=7,44 ; p<0,001)$, la dysthymie $(\mathrm{OR}=4,22 ; p<0,01)$, le trouble de panique $(\mathrm{OR}=3,47$; $p<0,01)$, l'anxiété généralisée ( $\mathrm{OR}=2,19 ; p<0,05)$, être un homme ayant subi une maltraitance ou une violence sexuelle pendant l'enfance $(\mathrm{OR}=21,01 ; p<0,01)$, être condamné pour des violences sexuelles $(\mathrm{OR}=7,12 ; p<$ $0,01)$ et la consommation de tabac $(\mathrm{OR}=2,93 ; p<0,01)$. Les covariables qui étaient significatives dans les analyses bivariées, mais ne l'étaient plus dans le modèle ajusté : vivre en ville, antécédents d'incarcération, condamnation pour infraction sur la législation de stupéfiants, consommation de drogues, spécifiquement consommation de cannabis. Parmi les pathologies psychiatriques, on retrouve l'agoraphobie, le TOC, le trouble de stress post-traumatique, les troubles psychotiques et le trouble de la personnalité antisociale.

Ce travail a permis d'évaluer pour la première fois la prévalence du risque suicidaire et d'identifier ses leviers dans une population carcérale dans un contexte socioculturel très particulier.

\section{Prévalence des délétions des gènes $p f h r p 2$ et pfhrp3 chez Plasmodium falciparum sur le plateau des Guyanes}

\author{
L. Berthelot ${ }^{1,2}$, Y. Lazrek ${ }^{1}$, B. Volney ${ }^{1}$, L. Musset ${ }^{1}$ \\ 1. Laboratoire de parasitologie, CNR paludisme, Pôle zones \\ endémiques, CC OMS surveillance des résistances aux anti- \\ paludiques, Institut Pasteur de la Guyane, 23 av. Pasteur, BP \\ 6010, Cayenne Cedex 97306, France ; 2. Faculté de méde- \\ cine, Université des Antilles, Fouillole, Pointe-à-Pitre \\ 97157, Guadeloupe, France \\ e-mail : berthelot.lena@gmail.com
}

Depuis 2009, l'Organisation mondiale de la santé (OMS) recommande de confirmer toute suspicion de paludisme avant un traitement antipaludique adapté en procédant à un diagnostic de certitude fondé sur la recherche des plasmodies par microscopie ou test de diagnostic rapide (TDR). Cette recommandation a conduit à un essor des TDR qui sont devenus des outils diagnostiques de choix dans les zones reculées aux infrastructures limitées. Ces TDR permettent notamment la détection de Plasmodium falciparum par la recherche de la protéine-2-riche en histidine (Pf-HRP2). Des parasites délétés pour le gène $p f h r p 2$, qui code cette protéine, ont cependant été décrits en Amazonie. Le risque est alors d'étiqueter à tort un accès à $P$. falciparum comme un accès à Plasmodium vivax et de le traiter par chloroquine alors que sur le plateau des Guyanes, 20 à $30 \%$ des $P$. falciparum sont chloroquinorésistants. En raison des flux migratoires et de la place des TDR dans cette région, une surveillance accrue de ces délétions est nécessaire.

Ainsi, des isolats collectés entre 2015 et 2017 en Guyane, au Guyana et au Venezuela ont été étudiés selon les recommandations de l'OMS afin d'estimer la prévalence des délétions au niveau des gènes pfhrp2/3. L'OMS préconise pour la caractérisation des parasites délétés une analyse des délétions totales et partielles des gènes $p f h r p 2$ et $p f h r p 3$, de leurs gènes adjacents et l'amplification d'un ou plusieurs autres gènes monocopies. 
Huit PCR analysant les deux parties, jonction exons 1-2 et exon 2 , des gènes $p f h r p 2$ et $p f h r p 3$ et leurs gènes adjacents ont ainsi été utilisées. Le protocole d'étude comprenait également une PCR en temps réel comme méthode semiquantitative permettant d'évaluer la qualité et la quantité de l'ADN des isolats étudiés afin de sélectionner les échantillons analysables.

Un total de 690 isolats a été étudié. Au Venezuela, 39,0\% (IC $95 \%$ : [28,1-49,9]) des parasites présentaient une délétion au niveau du gène $p$ fhrp 3 et 2,6\% (IC $95 \%:[0-6,8]$ ) au niveau de $p f h r p 2$. Au Guyana $(n=265)$ et en Guyane $(n=230)$, aucun parasite délété pour le gène $p f h r p 2$ n'a été mis en évidence et respectivement 3,0\%, IC $95 \%$ : [0,9\%$5,1 \%]$ et $0,5 \%$, IC $95 \%$ : [0-1,5] pour le gène pfhrp 3 .

Ces données robustes mettent pour la première fois en évidence des isolats délétés pour le gène $p f h r p 2$ au Venezuela et confirment leur absence en Guyane et au Guyana. Par conséquent, la surveillance reste de mise au Venezuela, mais les TDR fondés sur Pf-HRP2 restent des outils diagnostiques adaptés dans ces pays.

\section{Infection urinaire de l'adulte : prise en charge par les médecins généralistes en Guyane française}

\section{Y. Briquet, F. Djossou}

Unité des maladies infectieuses et tropicales, CHAR, Cayenne 97306, Guyane française

e-mail : yasmina.briquet@ch-cayenne.fr

Plusieurs recommandations sur le diagnostic et l'antibiothérapie des infections urinaires ont été successivement émises en France. Nous avons souhaité connaître le niveau d'adhésion des médecins généralistes guyanais à ces recommandations, et notamment celles de 2014.

Une enquête prospective s'est appuyée sur deux questionnaires : l'un décrivant les pratiques habituelles des médecins et l'autre analysant leur pratique effective sur la base de patients pris en charge pour infection urinaire.

Cent soixante-trois patients, dont $85,9 \%$ de femmes, ont été inclus par 54 des 96 médecins généralistes sollicités pour participer à l'étude (soit 56,3\% des médecins généralistes libéraux de Guyane). Parmi ceux-ci, 44,4\% avaient pris connaissance des nouvelles recommandations, et 55,6\% déclaraient s'y conformer avec la prescription de fosfomycine en cas de cystite aigue simple. La bandelette urinaire (BU) n'a été faite qu'à $27 \%$ de l'ensemble des patients. Sur les 88 examens cytobactériologiques des urines (ECBU) prescrits, 42 étaient conformes aux recommandations. Les molécules utilisées étaient adaptées dans $50 \%$ des cas, avec la prescription de fluoroquinolones à $46,7 \%$. Le principal germe identifié était Escherichia coli dans $67 \%$ des cas.
Une réévaluation clinique n'avait été faite que dans 19,6\% des cas. La démarche globale de prise en charge n'était conforme que pour $7,4 \%$ des patients.

Cette étude révèle un écart entre les pratiques des médecins généralistes de Guyane et leurs déclarations en matière de prise en charge des infections urinaires de l'adulte. Les déclarations et pratiques observées restent discordantes des recommandations de 2014, notamment en ce qui concerne la réalisation des BU, des ECBU, de l'échographie et de l'antibiothérapie. Des actions de formations sont nécessaires.

\section{Saturnisme pédiatrique sur la commune de Camopi, fleuve Oyapock, Guyane}

\author{
P. Cousin ${ }^{1,2}$, B. Guarmit ${ }^{1}$, E. Mosnier ${ }^{1}$, P. Brousse ${ }^{1}$, \\ E. Martin ${ }^{1,3}$
}

1. Service des Centres de prévention et de soins, CHAR, Cayenne 97306, Guyane française ; 2. Département universitaire de médecine générale, Université des Antilles, Fouillole, Pointe-à-Pitre 97157, Guadeloupe, France ; 3. Service de pédiatrie, CHAR, Cayenne 97306, Guyane française

e-mail : pauline.cousin@laposte.net

Le saturnisme pédiatrique en Guyane est connu depuis 2011. Son impact sanitaire n'a toutefois pas encore été évalué. Camopi est une commune à majorité amérindienne (Téko et Wayãpi), située sur le fleuve Oyapock à la frontière entre la Guyane et le Brésil. Elle est particulièrement impactée par le saturnisme. L'objectif de ce travail était de décrire les cas de saturnisme dans la population de Camopi, leur prise en charge médicale ainsi que d'éventuels cofacteurs carentiels associés.

Nous avons mené une étude observationnelle quantitative rétrospective monocentrique dans les centres délocalisés de prévention et de soins de Camopi et de Trois-Sauts (village appartenant à la commune de Camopi) auprès de tous les mineurs $(<18$ ans) ayant eu un dosage de plombémie supérieur à $50 \mu \mathrm{g} / \mathrm{l}$ entre 2012 et 2016 . Nous avons calculé des prévalences minimales fondées sur le nombre de diagnostics rapportés à l'effectif de population. Nous avons utilisé les données sociodémographiques et clinicobiologiques des dossiers médicaux et avons évalué le suivi clinicobiologique par rapport aux recommandations de la Haute Autorité de santé (HAS) chez les enfants de zéro à six ans.

Trente-neuf plombémies ont été dosées chez les 959 mineurs de la commune. Trente-quatre plombémies étaient supérieures au seuil de $50 \mu \mathrm{g} / \mathrm{l}$, dont sept à Camopi et 27 à Trois-Sauts. La prévalence minimale du saturnisme parmi toutes les demandes de plombémie sur la période était de $3,5 \%$ chez les moins de 18 ans de la commune et de $12,1 \%$ chez les moins de six ans à Trois-Sauts. La 
plombémie médiane était de 104,5 $\mu \mathrm{g} / \mathrm{l}$. Les plombémies médianes les plus élevées étaient retrouvées chez les filles de deux à dix ans $(116,5 \mu \mathrm{g} / \mathrm{l})$ et chez les garçons de 10 à 18 ans $(133 \mu \mathrm{g} / \mathrm{l})$. Peu de cas de saturnisme semblaient symptomatiques. Deux carences martiales associées ont été identifiées. Peu de données étaient disponibles concernant le suivi pédiatrique usuel. Deux mineurs sur 34 avaient bénéficié d'un examen systématique du nourrisson dans leur enfance. Nous n'avons pas retrouvé de renseignements sur le développement neurologique des enfants. Les courbes de taille étaient inexploitables dans 57 à $81 \%$ des cas selon l'âge. Chez les 17 enfants de zéro à six ans, quatre surveillances biologiques des plombémies étaient conformes aux recommandations de la HAS, et aucune surveillance clinique spécifique du saturnisme n'était renseignée.

La prévalence du saturnisme pédiatrique sur la commune de Camopi est alarmante et sous-évaluée. À Trois-Sauts, la prévalence du saturnisme à taux supérieur à $100 \mu \mathrm{g} / \mathrm{l}$ chez les enfants de zéro à six ans serait plus de 50 fois supérieure aux moyennes nationales. Peu d'anomalies clinicobiologiques ou de carences associées ont été mises en évidence. Ce constat est à nuancer au vu des insuffisances de suivi usuel et spécifique du saturnisme. L'optimisation du suivi et de la prise en charge des enfants est donc primordiale. Une collaboration pluridisciplinaire nous semble nécessaire afin de proposer des solutions concrètes d'éviction des facteurs causaux alimentaires et environnementaux.

\section{Pneumopathie aiguë communautaire de l'adulte immunocompétent et fièvre $\mathbf{Q}$ : connaissances, attitudes et perceptions des médecins généralistes de Guyane française}

\section{O. Denis ${ }^{1,2}$, J. Hurpez ${ }^{2}$, F. Djossou ${ }^{1}$, L. Epelboin ${ }^{1}$}

1. Unité des maladies infectieuses et tropicales CHAR, Cayenne 97306, Guyane française ; 2. Département universitaire de médecine générale, Université des Antilles, Fouillole, Pointe-à-Pitre 97157, Guadeloupe, France

e-mail : orianedenis1@gmail.com

En Guyane, la fièvre Q (FQ) est la cause de 24 à $38 \%$ des pneumopathies aiguës communautaires (PAC) hospitalisées. Ainsi, toute pneumopathie est d'emblée traitée par doxycycline en plus de l'antibiothérapie antipneumococcique habituellement recommandée. Les médecins généralistes $(\mathrm{MG})$ étant en première ligne dans la prise en charge de la FQ, comme des PAC, l'objectif de cette étude était d'évaluer les connaissances et perceptions des MG de Guyane sur ces deux sujets liés.

Un questionnaire portant sur les connaissances et les pratiques vis-à-vis des PAC et de la FQ a été distribué aux MG de Guyane entre février et juillet 2017. Les MG ont été iden- tifiés via le conseil départemental de l'ordre des médecins et les pages jaunes. Ont été exclus de l'étude les MG exerçant en milieu hospitalier. Concernant les connaissances pour la FQ et les PAC, les caractéristiques des médecins prescrivant la doxycycline ont été comparées aux autres, puis les connaissances des médecins de l'île de Cayenne ont été comparées à celles du reste de la Guyane.

Parmi les 113 MG identifiés comme exerçant sur le territoire sur la période d'étude, 54 (47,8\%) ont répondu au questionnaire. Ils voyaient moins de cinq PAC par mois pour 90,7 \% d'entre eux (49/54), et 59,2\% (32/54) prescrivaient systématiquement une radiographie de thorax, $64,8 \%$ (35/54) prescrivaient de l'amoxicilline chez un sujet jeune sans antécédent particulier et 77,8 \% (41/54) de l'amoxicilline + acide clavulanique chez un sujet âgé souffrant d'insuffisance cardiaque congestive. Les MG les plus jeunes $(<40$ ans) étaient significativement plus à jour dans le traitement des PAC des sujets jeunes $(p=0,014)$ ou des sujets âgés $(p=0,04)$. En ce qui concerne la FQ, $37 \%$ affirmait en voir au moins une par mois et $26 \%$ plus d'une tous les six mois. Seuls $26 \%$ d'entre eux savaient que Coxiella burnetii est la première cause documentée de PAC en Guyane. Si $96 \%$ savaient que la sérologie est l'examen de référence, $46 \%$ pensaient qu'il fallait la réaliser entre $\mathrm{j} 6$ et j14 et $19 \%$ entre j15 et j21. Seuls $22 \%$ d'entre eux associaient la doxycycline au traitement antipneumococcique pour le traitement d'une PAC du sujet jeune. Les facteurs associés à la prescription de doxycycline étaient le fait d'exercer sur l'île de Cayenne pour le traitement de la PAC du sujet jeune et d'avoir moins de 40 ans (pour les médecins) dans le traitement de la PAC du sujet âgé. Les MG de l'île de Cayenne savaient qu'une échographie cardiaque est nécessaire en cas de diagnostic positif, que le diagnostic passait par la répétition des sérologies et que le traitement d'une PAC du sujet jeune doit comprendre de la doxycycline. 59,3\% (32/54) des MG de notre étude se sentaient mal informés sur la FQ et $98,1 \%(53 / 54)$ seraient intéressés par des formations.

Les connaissances et pratiques des MG de Guyane n'étaient généralement pas en concordance avec les dernières recommandations sur la prise en charge des PAC, résultat déjà rapporté dans la littérature. La FQ était encore peu connue des MG malgré son poids en Guyane, ce qui témoigne d'un défaut de partage des connaissances entre hôpital et ville malgré les nombreuses publications sur le sujet en Guyane. On constate néanmoins que sur l'île de Cayenne, les MG semblent plus sensibilisés et à jour sur certains points du traitement ou des examens complémentaires que dans le reste de la Guyane. Des actions de formations permettraient de sensibiliser de nombreux acteurs pour une meilleure prise en charge des PAC et de la FQ en ambulatoire. 


\section{Évaluation de l'accès aux soins des demandeurs d'asile et des réfugiés originaires du Moyen-Orient en Guyane}

\section{F. Diab ${ }^{1,2}$, G. Egmann $^{3}$, J. Marlier ${ }^{3}$}

1. Service de la PASS (Permanence d'accès aux soins de santé) de Cayenne, Guyane française ; 2. Département de médecine générale, Université des Antilles, Fouillole, Pointe-à-Pitre 97157, Guadeloupe, France ; 3. Service des urgences adultes du CHAR, Cayenne, Guyane française

e-mail : floriane.diab@gmail.com

La guerre civile syrienne est responsable de la plus grande crise migratoire et humanitaire depuis la Seconde Guerre mondiale. La Guyane n'est pas épargnée par cet afflux de migrants en provenance du Moyen-Orient. Nous avons constaté une difficulté de prise en charge de ces patients du fait de la barrière de la langue. L'objectif principal de cette étude était d'évaluer l'accès aux soins aigus des demandeurs d'asile et des réfugiés en provenance du Moyen-Orient.

Nous avons réalisé une étude transversale, rétrospective. Les patients demandeurs d'asile ou réfugiés originaires du Moyen-Orient arrivés en Guyane entre janvier 2014 et octobre 2016 constituaient notre critère d'inclusion. L'échantillonnage a été réalisé selon un mode boule de neige et selon les lieux de rencontre. Les données étaient recueillies sous forme d'entretien individuel.

Quarante-cinq patients ont été inclus sur une population estimée de 150 personnes selon l'OFPRA. Soixante et onze pour cent étaient originaires de Syrie, $20 \%$ étaient originaires de Palestine, $10 \%$ étaient des enfants nés au cours du parcours migratoire (Brésil et Venezuela). Le pic d'immigration était en 2016. La moyenne d'âge de notre population était de 23,6 ans. Les deux tranches d'âge les plus représentées étaient celles des $2-10$ et $35-44$ ans. $70,5 \%$ de notre population correspondaient à des familles avec enfants. Tous les adultes de notre population avaient eu accès à l'éducation, et $37 \%$ avaient fait des études supérieures. Quatre-vingt-huit pour cent avaient un parcours migratoire long (transit par plus de deux pays). Les pathologies étaient similaires à celles rencontrées habituellement en Europe. Tous les patients ayant présenté une pathologie aiguë (majoritairement des pathologies infectieuses [36,4\%] et des pathologies dermatologiques $[21,2 \%])$ ont eu accès aux soins. Deux patients ont présenté des dégradations de pathologies chroniques, dont une grave. Aucun décès n'a été enregistré. La principale difficulté de prise en charge a été la barrière de langue avec $45 \%$ des patients arabophones stricts. Cinquante pour cent des communications avec le personnel soignant étaient jugées comme impossibles à établir. Lorsque le patient recevait une bonne information sur le parcours de soins et les démarches administratives à effectuer (communication entre le médecin et le patient de qualité), il avait significativement plus de chance d'avoir accès au traitement $(p=0,019)$.

Notre étude montre que la barrière de langue constitue un véritable obstacle à l'accès aux soins et est responsable d'une aggravation des problèmes de santé. Si aucun décès n'a été enregistré durant la période d'inclusion de l'étude, il a néanmoins été constaté des dégradations de pathologies chroniques non traitées et/ou non suivies qui auraient pu être évitables. La création d'un service d'interprétariat au centre hospitalier de Cayenne, véritable carrefour migratoire, est une nécessité, que cela soit pour nos patients issus du Moyen-Orient, que pour ceux originaires de pays anglophones, hispanophones, lusophones, sinophones, créolophones rencontrés dans notre pratique quotidienne. Il est à craindre que ces populations, fragilisées par leur statut de réfugié ou de migrant, ne puissent pas bénéficier des mêmes chances de prise en charge que les autres.

\section{Contage varicelleux chez une femme enceinte en demande d'asile, en Guyane française}

\section{F. Diab ${ }^{1,2,3}$, A. Monthieux ${ }^{3}$}

1. Service de la PASS (Permanence d'accès aux soins de santé) de Cayenne, Guyane française ; 2. Département de médecine générale, Université des Antilles, Fouillole, Pointe-à-Pitre 97157, Guadeloupe, France ; 3. Urgences gynécologiques de la Maison de la femme de la mère et de l'enfant, Fort-de-France, Martinique

e-mail : floriane.diab@gmail.com

C'est au cours du recueil de données pour ma thèse que je fais la rencontre de Mme A. et de sa famille, ayant fui la Syrie suite au conflit armé sévissant depuis 2011. Lors de mon entretien, je diagnostique une varicelle chez son fils de cinq ans. Mme A. est alors enceinte de 26 semaines d'aménorrhées et dit ne jamais avoir eu la varicelle dans l'enfance. La prise en charge de la varicelle chez la femme enceinte ne faisant pas l'objet d'un consensus national, ce travail s'attache à étudier la littérature et les recommandations au niveau international afin d'analyser les différentes pratiques et à mettre en avant le rôle essentiel du médecin traitant dans la prévention de la varicelle chez la femme enceinte. Nous nous intéressons également à la procédure de demande d'asile et aux aides sociales disponibles. Enfin, nous développons les possibilités de communication entre le médecin traitant et les patients allophones.

\section{Création d'une chaîne de vidéos traitant de pathologies guyanaises}
A. Fournet ${ }^{1}$, F. Schreiber ${ }^{1}$, J. Hurpez ${ }^{1}$, L. Epelboin ${ }^{2}$, P. Couppie $^{2}$, M. Demar ${ }^{2}$, C. Marty ${ }^{3}$, F. Djossou ${ }^{2}$, F. Niemetzky ${ }^{1,2}$ 
1. Département de médecine générale, Université des Antilles, Fouillole, Pointe-à-Pitre 97157, Guadeloupe, France ; 2. CHAR, Cayenne 97306, Guyane française ; 3. Croix-Rouge française, Cayenne, Guyane française

e-mail : alice.fournet1@gmail.com

La Guyane est un « désert médical », et son accroissement démographique est l'un des plus importants de France. Il existe une inadéquation entre les besoins et l'offre en formation sur les pathologies spécifiques à la région. La Guyane possède une richesse en matière de recherche scientifique et d'exercice clinique que nos confrères ignorent. De multiples travaux de recherches sont en train de naître sur le territoire guyanais. À travers un travail universitaire original, trois internes de médecine générale ont créé un outil pédagogique innovant sur des problématiques locales passionnantes. Ils ont créé une chaîne YouTube " La médecine en Guyane » sur laquelle a été lancée le 15 juin une première série de six épisodes portant sur des pathologies tropicales retrouvées en Guyane : la dengue, la toxoplasmose amazonienne, la leishmaniose cutanée, la fièvre $Q$, la leptospirose et les envenimations par morsure de serpent. Notre objectif est de valoriser la médecine guyanaise et ses particularités afin d'augmenter l'attractivité locale. Les pathologies étudiées font partie de la pratique courante du médecin généraliste en Guyane. De plus, l'éducation à la santé est l'une des missions du médecin généraliste. Nous voulons délivrer, à nos confrères, un aperçu attrayant de la diversité des pathologies rencontrées et leur donner ainsi l'envie de découvrir la richesse de la pratique médicale en Guyane. De plus, il s'agit d'un territoire propice à la mise en place de stratégies innovantes en matière de promotion et d'éducation pour la santé.

\section{Épidémiologie des intoxications aiguës pédiatriques en Guyane 2013-2015}

\section{A. Fournet ${ }^{1,2}$, J. Langrand ${ }^{3}$, M. Nacher ${ }^{4}$, N. Elenga ${ }^{5}$, F. Henaff ${ }^{2,5}$}

1. Département universitaire de médecine générale, Université des Antilles, Fouillole, Pointe-à-Pitre 97157, Guadeloupe, France ; 2. Service d'accueil des urgences, CHAR, Cayenne 97306, Guyane française ; 3. Centre antipoison toxicovigilance, Hôpital Fernand Widal, Paris ; 4. Centre d'investigation clinique Antilles Guyane, INSERM CIC 1424, CHAR, Cayenne 97306, Guyane française ; 5. Service de pédiatrie, CHAR, Cayenne 97306, Guyane française

e-mail : alice.fournet1@gmail.com

Chez les enfants en bas âge, les intoxications aiguës sont le plus souvent accidentelles du fait de l'ingestion de produits laissés à leur portée. Leurs caractéristiques actuelles ne sont pas connues en Guyane française.
Nous avons réalisé une étude épidémiologique rétrospective des cas pédiatriques d'ingestion de produits toxiques ayant donné lieu à une consultation dans les trois hôpitaux et 17 centres délocalisés de prévention et de soins (CDPS) de Guyane et/ou ayant été signalés aux centres antipoisons, entre le $1^{\text {er }}$ janvier 2013 et le 31 décembre 2015. Les produits toxiques étaient des xénobiotiques (produits étrangers à l'organisme) incluant produits chimiques, ménagers, pharmaceutiques, cosmétiques, végétaux, tabac, alcool.

Durant cette période, 662 cas ont été recensés chez des enfants de moins de dix ans. L'âge moyen était de 2,5 ans $( \pm 1,6)$. Il s'agissait généralement de mono-intoxications ( $n$ $=617 ; 97,47 \% ; p=0,002)$, plus fréquemment liées à un défaut de perception du risque $(n=337 ; 89,4 \% ; p<$ 0,001 ). Trois classes de produits ingérés (ménagers, chimiques et pharmaceutiques) ont été impliquées majoritairement, quel que soit l'âge $(n=542 ; 81,6 \% ; p<0,001)$. Le lieu de consultation n'était pas renseigné pour 205 enfants. Ces cas ont fait l'objet d'un appel aux centres antipoison, mais n'ont pas été retrouvés parmi les cas issus des différentes structures de soins de Guyane. Sur les 457 consultations retrouvées, 335 enfants $(73,3 \%)$ sont rentrés à leur domicile, 88 (19,3\%) ont été hospitalisés. Les hospitalisations étaient plus souvent liées à l'ingestion de produits chimiques $(n=$ $26 ; 29,6 \% ; p<0,001)$. Dans les centres de santé des communes isolées, plus de la moitié des cas inclus $(n=17$, $65,4 \%$ ) étaient liés à l'ingestion d'essence ou de raticides.

Les intoxications aiguës étaient fréquentes et le plus souvent liées à des comportements à risque. Parmi les cas observés, certains produits sont interdits en France tels que paraquat, malathion et cannabis. Cela a mis en évidence la nécessité d'accroître la prévention et de développer des stratégies adaptées au contexte guyanais.

\section{Mort fotale in utero : épidémiologie et facteurs de risque de récidive dans l'Ouest guyanais, à propos de 370 cas}

G. Frossard ${ }^{1}$, V. Lambert ${ }^{2}$, G. Carles ${ }^{2}$, L. Pomar $^{2}$, J. Missemer $^{2}$, A. Jolivet ${ }^{3,4}$, M. Dreyfus ${ }^{5}$

1. Service de gynécologie obstétrique et médecine de la reproduction, CHU de Caen, avenue de la Côte de Nacre, 14033 Caen Cedex, France ; 2. Service de gynécologie et obstétrique, Centre hospitalier de l'Ouest guyanais, 16 bld du Général Leclerc, BP 245, 97393 Saint Laurent du Maroni, Guyane française ; 3 . Pôle de santé publique, Centre hospitalier de l'Ouest guyanais, 16 boulevard du Général Leclerc, BP 245, 97393 Saint Laurent du Maroni, Guyane française ; 4. Sorbonne Universités, UPMC Université Paris 06, INSERM, Institut Pierre Louis d'épidémiologie et de santé publique (IPLESP UMRS 1136, Équipe de recherche en épidémiologie sociale), 75012 Paris, France ; 5. Université 
de médecine Caen Basse-Normandie, CHU de Caen, av. de la Côte de Nacre, 14033 Caen Cedex, France

e-mail : guillemettefrossard@yahoo.fr

Il s'agit de décrire les aspects épidémiologiques, cliniques et étiologiques des morts fotales in utero (MFIU) ou de leur récidive en Guyane française, dans un contexte géopolitique particulier, des moyens de pays à haut revenu dans une zone géographique comprenant une population variée et un accès aux soins très inégalitaire.

Une étude observationnelle rétrospective a été menée dans une maternité de type IIB à Saint-Laurent-du-Maroni sur la survenue des MFIU entre 1998 et 2016. Les données ont été relevées à partir de dossiers informatisés et papiers. Les données des patientes ayant une récidive de MFIU ont été comparées aux autres patientes sans récidive.

Trois cent soixante-dix cas de MFIU ont été identifiés, soit 9,6 cas pour 1000 naissances. La principale cause était placentaire pour près de la moitié des cas dont $31 \%$ d'hématome rétroplacentaire touchant les MFIU tardives (soit $67 \%$ des MFIU), contrairement aux causes infectieuses qui concernaient les MFIU précoces (soit $33 \%$ des MFIU), $p<0,001$. Il y a eu 47 cas de récidives, soit $14 \%$, la principale étiologie était la cause placentaire (60\% des cas).

Trois facteurs de risque de récidive ont été identifiés en analyse multivariée : la grande multiparité, l'antécédent de prématurité et l'anémie pendant la grossesse, respectivement $\mathrm{OR}=3,38[1,55-7,35], \mathrm{OR}=3,73[1,61-8,66], \mathrm{OR}=2,85$ $[1,39-5,82]$.

L'identification des femmes à risque de MFIU semble primordiale et notamment celles pouvant faire l'objet d'une prévention lors d'une grossesse en cours ou ultérieure.

\section{Évaluation d'un test d'immunocapture IgA maison pour le diagnostic du virus Zika}

A. Guérard ${ }^{1,2}$, D. Rousset ${ }^{1}$

1. Institut Pasteur de Guyane, CNR arbovirus, virus des infections respiratoires et Hantavirus ; 2. Collège sciences de la santé, UFR des sciences médicales, Université de Bordeaux

e-mail : alexxguerard@gmail.com

Dans un contexte d'épidémie de Zika, un suivi sérologique des femmes enceintes est important afin de surveiller la survenue d'infection en cours de grossesse et de détecter d'éventuelles transmissions, voire de complications fœtales. Malheureusement, ce suivi n'est pas toujours réalisé, et l'interprétation sérologique est délicate avec des IgM pouvant rester détectables dans le sang plus de 300 jours après les symptômes. Le développement d'outils complémentaires est donc nécessaire.
Deux panels ont été utilisés pour valider les performances analytiques d'un test d'immunocapture IgA (AAC-Elisa) maison (technique immunoenzymatique qui permet de mettre en évidence un complexe antigène-anticorps à l'aide d'une réaction enzymatique) en tant qu'outil supplémentaire de diagnostic et de datation de l'infection chez la femme enceinte. Pour l'évaluation des performances analytiques, 199 sérums de patients prélevés entre $\mathrm{j} 3$ et j20 après le début de symptômes évocateurs d'une infection arbovirale ont été testés, dont 90 sérums étaient positifs pour le virus Zika. Pour l'étude de la cinétique des IgA, 300 prélèvements séquentiels de patients ayant présenté une infection confirmée par RT-PCR pour le virus Zika ont été analysés.

Ce test IgA a montré une meilleure spécificité (79\%), associée à une moins bonne sensibilité (67\%) [surtout en début et à distance de l'infection] que le test IgM pour le diagnostic d'infection par le virus Zika. Il se caractérise surtout par une fenêtre de positivité des IgA beaucoup plus étroite $(<40$ jours $v s>300$ jours pour les IgM).

La technique d'immunocapture IgA constitue ainsi un outil diagnostic complémentaire pour le diagnostic sérologique de l'infection à virus Zika permettant, en cas d'IgM antivirus Zika positives, d'aider à apprécier le caractère récent ou non de l'infection.

\section{Prise en charge initiale du syndrome coronarien $\mathbf{S T}+$ en milieu isolé : l'exemple de la Guyane française}

M. Horellou ${ }^{1,2}$, H. Kallel $^{3}$, C. Bonnefoy ${ }^{1}$, G. Egmann ${ }^{1}$

1. Service d'accueil des urgences, CHAR, Cayenne 97306, Guyane française ; 2. Département de médecine générale, Université des Antilles, Fouillole, Pointe-à-Pitre 97157, Guadeloupe, France ; 3. Service de réanimation, CHAR, Cayenne 97306, Guyane française

e-mail : marion.horellou@ch-cayenne.fr

La Guyane est un département français isolé d'Amérique $\mathrm{du}$ Sud où l'accès aux soins est souvent plus difficile qu'en métropole : le territoire ne dispose pas de cardiologie interventionnelle. La thrombolyse, avant évacuation sanitaire (evasan), est donc l'unique traitement étiologique disponible pour la prise en charge du syndrome coronarien aigu $\mathrm{ST}+$ (SCA ST+). Malgré cette singularité, la prise en charge du SCA ST+ n'a jamais été étudiée sur le territoire. L'objectif principal de l'étude était de décrire la prise en charge initiale du SCA ST+ en Guyane entre le $1^{\text {er }}$ janvier 2014 et le 31 décembre 2016 et de la comparer aux recommandations européennes et aux pratiques métropolitaines.

Il s'agissait d'une étude rétrospective et monocentrique menée au centre hospitalier Andrée-Rosemon, seul établissement du département disposant d'une unité de soins 
intensifs cardiologiques. Les patients pris en charge pour un SCA ST+ en Guyane française évoluant depuis moins de 24 heures, identifiés parmi les patients admis au CHAR et pris en charge aux urgences, en USIC, en réanimation ou ayant bénéficié d'une évacuation sanitaire intra- ou extradépartementale par le Smur ont été inclus. Le recueil a concerné les données épidémiologiques, la symptomatologie initiale et le mode de recours des patients ainsi que les principaux éléments de leur prise en charge médicale initiale, notamment les délais de réalisation d'ECG, de thrombolyse et d'évacuation sanitaire. Le devenir à 30 jours des patients a également été étudié. Les variables continues ont été décrites en calculant les médianes et interquartiles et les variables binomiales à l'aide de pourcentages. Les variables ont été comparées en analyse bivariée en utilisant un test du Chi-2 pour les variables binomiales et les tests de Student et Wilcoxon pour les variables continues. Le seuil de significativité a été fixé à $5 \%$. Les valeurs manquantes lors du recueil n'ont pas été intégrées à l'analyse statistique. Les données de l'étude ont été comparées aux résultats de métropole grâce aux données extraites des registres spécialisés FAST MI 2015 (national) et e-MUST (île-de-France). Nos délais de prise en charge ont été comparés aux objectifs définis par la Société européenne de cardiologie en 2017. Dans une seconde analyse, nous avons comparé nos résultats chez les patients ayant ou non bénéficié d'une prise en charge Smur initiale.

Ont été inclus 111 patients pris en charge pour un SCA ST + de moins de 24 heures. La moyenne d'âge était de 58 ans $[45 ; 71]$. Le délai de consultation médian était de 1 heure et 44 minutes IQ [0 heure 49 minutes ; 3 heures 53 minutes]. Le taux de thrombolyse était de $69,4 \%$, le taux de revascularisation de $52,7 \%$ ( $97 \%$ en Île-de-France) et le délai douleur-thrombolyse de 3 heures IQ [1 heure 44 minutes ; 4 heures 44 minutes]. Soixante-neuf pour cent des patients ont bénéficié d'une evasan dans un délai médian de 35 heures et 48 minutes IQ [20 heures 31 minutes ; 53 heures 40 minutes]. Le taux de mortalité à 24 heures était de 7,2\% contre $2,6 \%$ en métropole. Le taux d'appel au Samu était de 50,9 \% (76 \% en Île-de-France).

La prise en charge initiale par le Smur (34,3\% des patients) permettait une augmentation du taux de thrombolyse $(86,1$ contre $61,3 \%, p=0,008)$, une réduction des délais de consultation ( 60 contre 126 minutes, $p \leq 0,001$ ) et de thrombolyse (120 contre 231 minutes, $p \leq 0,001$ ).

La prise en charge initiale du SCA ST+ en Guyane n'est comparable ni aux recommandations européennes ni aux pratiques françaises : le taux de thrombolyse est trop faible et les délais de prise en charge et d'evasan trop longs. Si une sensibilisation de la population guyanaise ainsi qu'une optimisation des pratiques médicales locales pourraient l'améliorer, la gestion du SCA ST+ en Guyane française ne pourra pas être comparable à celle des autres départements français tant que le territoire guyanais ne disposera pas de son propre plateau de coronarographie interventionnelle.

\section{Caractéristiques épidémiologiques et cliniques des gastroentérites aiguës non invasives de l'enfant à l'hôpital de Cayenne}

J. Hurpez ${ }^{1,2}$, M. Nacher ${ }^{3}$, B.-M. Imbert ${ }^{4}$, N. Elenga ${ }^{1}$, C. Gras-Leguen ${ }^{5}$, F. Henaff ${ }^{6}$

1. Service de pédiatrie du Centre hospitalier Andrée Rosemon et Département universitaire de médecine générale, Université des Antilles, Fouillole, Pointe-à-Pitre 97157, Guadeloupe, France ; 2. Département de médecine générale, Université des Antilles, Fouillole, Pointe-à-Pitre 97157, Guadeloupe, France ; 3. Centre d'investigation clinique Antilles Guyane, INSERM CIC 1424, CHAR, Cayenne 97306, Guyane française ; 4. Laboratoire de virologie du CHU de Nantes, France ; 5 . Service de pédiatrie du CHU de Nantes, France ; 6. Service de pédiatrie du CHAR, Cayenne 97306, Guyane française

e-mail : julian.hurpez@gmail.com

L'agent étiologique le plus souvent en cause dans les GEA sévères chez le nourrisson et l'enfant de moins de cinq ans est le rotavirus. Aucune étude concernant les gastroentérites aiguës (GEA) en Guyane n'a jamais été réalisée. L'objectif de ce travail était de décrire l'épidémiologie des GEA de l'enfant à Cayenne et la proportion de rotavirus.

Nous avons réalisé une étude épidémiologique, descriptive, prospective et rétrospective à l'hôpital de Cayenne entre août 2016 et août 2017. La phase prospective consistait à inclure tous les enfants consultant aux urgences ou hospitalisés pour diarrhée non glairosanglante dans le cadre d'une gastroentérite aiguë. Lors de cette phase, pour chaque enfant inclus, un prélèvement de selles était réalisé pour analyse virologique, et le médecin investigateur devait remplir un CRF (clinical research form). Y étaient notifiés l'âge, le sexe, l'origine ethnique du patient et l'éventuelle vaccination antérieure de l'enfant contre le rotavirus. D'un point de vue clinique, il renseignait notamment sur le niveau de déshydratation de l'enfant (évalué par rapport à la perte de poids de l'enfant), la température maximum, la présence ou non de vomissements, l'état de la diarrhée. Enfin, il renseignait sur la thérapeutique adoptée et notamment sur une éventuelle réhydratation intraveineuse. La phase rétrospective consistait à recueillir tous les cas de gastroentérites aiguës ayant entraîné une consultation aux urgences du CHAR ou une hospitalisation dans le service de pédiatrie du CHAR entre août 2016 et août 2017.

Sur la période d'étude, 805 cas de GEA ont été recensés. L'âge moyen était de 2,9 ans $( \pm 3,26)$. La tranche d'âge la plus représentée était celle entre un et quatre ans (49,94\%). 
Le sex-ratio était de 1,29. Un pic de consultations pour GEA a été atteint au mois de mars $(n=109)$. Des vomissements étaient associés à la diarrhée dans 79,8\% des cas. Les enfants de plus de cinq ans présentaient moins de selles et des symptômes moins fréquents $(p<0,05)$. Le pourcentage de déshydratation était plus important chez les un à quatre ans $(3,7 \pm 2,6 \% ; p<0,05)$. Les enfants de moins d'un an ont été plus souvent hospitalisés $(15,2 \% ; p=0,003)$. Le rotavirus a été mis en évidence dans $29,9 \%$ des cas. Le pourcentage de déshydratation était plus important en cas de GEA à rotavirus $(4,3$ vs $2,6 \% ; p=0,025)$. Le taux d'enfants réhydratés par voie IV et hospitalisés $(34,8$ vs $21,7 \%$; $p$ non significatif) était plus élevé en cas de GEA à rotavirus.

Cette première étude a permis d'exposer les caractéristiques épidémiologiques et cliniques des GEA de l'enfant en Guyane et de mettre en évidence les virus circulants.

\section{Intérêt du dosage de la CRP dans la distinction précoce entre la dengue et la leptospirose en Guyane, une étude cas témoin appariée}

P. Le Turnier ${ }^{1}$, T Bonifay ${ }^{2}$, E. Mosnier ${ }^{1,3,4}$, R Schaub ${ }^{2}$, D Blanchet ${ }^{5}$, A Jolivet ${ }^{6}$, Magalie Demar ${ }^{4,5}$, M. Nacher ${ }^{2,4}$, P Bourhy $^{7}$, F. Djossou ${ }^{1,4}$, L. Epelboin ${ }^{1,4}$

1. Unité des maladies infectieuses et tropicales, CHAR, Cayenne 97306, Guyane française ; 2. Centre d'investigation clinique (CIC INSERM 1424), CHAR, Cayenne 97306, Guyane française ; 3. Service des CDPS, CHAR, Cayenne 97306, Guyane française ; 4. Equipe EA 3593, Ecosystèmes amazoniens et pathologies tropicales, Université de la Guyane, Cayenne, Guyane française ; 5. Laboratoire hospitalo-universitaire de parasitologie mycologie, CHAR, Cayenne 97306, Guyane française ; 6. Santé publique, Centre hospitalier de l'Ouest guyanais, SaintLaurent du Maroni ; 7. Institut Pasteur, CNR de la Leptospirose, Paris

e-mail : paul.leturnier@gmail.com

La leptospirose et la dengue sont des pathologies tropicales souvent difficiles à différencier. Un retard diagnostique et à l'initiation de l'antibiothérapie au cours de leptospirose peut s'avérer préjudiciable. L'objectif de ce travail était d'étudier les facteurs prédictifs entre la leptospirose et la dengue à l'admission.

Il s'agit d'une étude observationnelle rétrospective castémoin, réalisée en Guyane. Les patients atteints de leptospirose (test de microagglutination et/ou PCR) entre 2007 et 2014 (cas) ont été comparés à des patients atteints de dengue (Ag NS1 positif) de l'épidémie de 2013 (trois témoins par cas, appariés sur l'âge). Les données recueillies à l'admission ont été comparées par analyse bivariée appariée puis multivariée. La performance diagnostique des facteurs identifiés a été conduite par analyse de courbe ROC.

L'étude a porté sur 72 cas de leptospirose et 216 cas de dengue. Les critères démographiques et cliniques associés à la leptospirose étaient le sexe masculin et la toux. La présence d'un exanthème, d'un purpura, de myalgies, de céphalées était associée à la dengue. Les critères biologiques les plus discriminants en faveur de la leptospirose étaient : CRP $>50 \mathrm{mg} / 1$, leucocytes $>10 \mathrm{G} / \mathrm{l}$, créatinine $>120 \mu \mathrm{mol} / \mathrm{l}$, bilirubine totale $>20 \mu \mathrm{mol} / \mathrm{l}, \mathrm{CPK}>2 \mathrm{~N}$, hémoglobine $<10 \mathrm{~g} /$ dl. Un taux de leucocytes $<4 \mathrm{G} / 1$ et de polynucléaires neutrophiles $<1,5 \mathrm{G} / 1$ était associé à la dengue. Après analyse multivariée, la CRP $>50 \mathrm{mg} / \mathrm{l}(\mathrm{OR}: 141,3 ;[48,7-410,1])$ et la bilirubine totale $>20 \mu \mathrm{mol} / \mathrm{l}(\mathrm{OR}: 7,6 ;[1,6-35,9])$ étaient les facteurs associés de façon indépendante à la leptospirose. La sensibilité, la spécificité, les valeurs prédictives positive et négative du seuil de CRP $>50 \mathrm{mg} / \mathrm{l}$, étaient de 0,89 , de 0,95 , de 0,87 et de 0,96 respectivement. L'ajout de la bilirubine totale n'améliorait pas les performances de la CRP prise isolément.

La CRP était un marqueur sensible et spécifique pour discriminer la leptospirose de la dengue. Face à un syndrome dengue-like au retour ou en zone d'endémie, la CRP pourrait aider le clinicien à reconnaître une leptospirose et à débuter ainsi une antibiothérapie précoce. Ce marqueur nécessitera d'être validé dans de futures cohortes prospectives.

\section{Couverture vaccinale des enfants d'âge préscolaire admis au centre hospitalier de Cayenne en 2017}

C. Marre $^{1}$, A. Lucarelli ${ }^{2}$, N. Elenga ${ }^{3}$

1. Département universitaire de médecine générale, Université des Antilles, Fouillole, Pointe-à-Pitre 97157, Guadeloupe, France ; 2. Hôpital de jour adulte, consultations spécialisées, CHAR, Cayenne 97306, Guyane française ; 3. Service de médecine et chirurgie pédiatrique, CHAR, Cayenne 97306, Guyane française

e-mail : marrececile@gmail.com

Assurer la vaccination en Guyane est d'une importance capitale en raison de la forte croissance démographique et de l'accès aux soins limité. Cet accès aux soins limité est le résultat d'un isolement et d'une précarité élevés. Le but de cette étude était de décrire la couverture vaccinale (CV) des enfants de moins de trois ans à Cayenne et de déterminer les facteurs socio-économiques qui pourraient influencer le statut vaccinal ainsi que les causes du refus du vaccin.

Il s'agit d'une étude épidémiologique, prospective, transversale et monocentrique, réalisée du $1^{\mathrm{er}}$ janvier au $1^{\mathrm{er}}$ mai 2017. La population d'étude était constituée de tous les enfants en âge préscolaire se présentant au centre hospitalier 
de Cayenne. L'absence du carnet de santé entraînait l'exclusion de l'étude. Le recueil de données a été effectué à l'aide d'un questionnaire que les médecins devaient remplir au sein du service de pédiatrie ainsi qu'aux urgences pédiatriques. La CV a été estimée par la proportion de personnes ayant une immunisation complète pour 13 vaccins (dont quatre étaient obligatoires).

Cent quatre-vingt-quatre questionnaires ont pu être renseignés à partir des données des enfants identifiés. La CV était de $70 \%$ pour le vaccin pentavalent (diphtérie, tétanos, poliomyélite, Haemophilus influenzae et coqueluche), de $46 \%$ pour la fièvre jaune, de $70 \%$ pour l'hépatite $\mathrm{B}$, de $50 \%$ pour le vaccin ROR et le pneumocoque et de $7 \%$ pour le méningocoque. Aucun lien n'a été trouvé entre le mode de garde de l'enfant, son lieu de naissance ou la catégorie socioprofessionnelle des parents et le statut vaccinal. Les raisons principales de la non-vaccination pour tous les vaccins étaient les problèmes de transport et l'absence de proposition du vaccin par le médecin traitant. Cette faible couverture pour le vaccin antiamaril, pourtant obligatoire en Guyane et disponible chez le médecin libéral, montre la nécessité de former les médecins généralistes aux techniques et aux stratégies vaccinales les plus récentes et de les informer sur les obligations et les recommandations du calendrier vaccinal et sur les objectifs poursuivis. Il y avait une pénurie franche de vaccins BCG sur la période d'étude. Il est important de noter que les refus étaient peu nombreux, les parents guyanais étant assez favorables à la vaccination.

La couverture vaccinale des enfants de moins de trois ans était médiocre en Guyane française et nécessite la mise en place des mesures pour l'améliorer. Il est indispensable de sensibiliser davantage les professionnels de santé et la population dans son ensemble aux bienfaits individuels et collectifs de la vaccination.

\section{Syndrome inflammatoire de reconstitution immunitaire (IRIS) associé à Histoplasma capsulatum var. capsulatum (Hcc) chez des patients infectés par le VIH en Guyane française : étude de cas sur une période de $\mathbf{2 0}$ ans et révision de la littérature}

A. Melzani ${ }^{1,2}$, R. De Reynal De Saint $\mathrm{Michel}^{3}$, B. Ntab ${ }^{4}$, F. Djossou ${ }^{1,2}$, L. Epelboin ${ }^{1,2}$, M. Nacher ${ }^{2,5}$, D. Blanchet ${ }^{2,6}$, M. Demar ${ }^{2,6}$, P. Couppie ${ }^{2,3}$, A. Adenis ${ }^{2,5}$

1. Unité des maladies infectieuses et tropicales, Centre hospitalier de Cayenne, Cayenne, Guyane française ; 2. Equipe EA3593, Ecosystèmes amazoniens et pathologie tropicale, Université de Guyane, Cayenne, Guyane française ; 3. Service de dermatologie-vénérologie, Centre hospitalier de Cayenne, Cayenne, Guyane française ; 4. Département d'information médicale, Centre hospitalier de l'Ouest guya- nais, Saint Laurent du Maroni, France ; 5. Centre d'investigation clinique Antilles Guyane, Inserm CIC 1424, Centre hospitalier de Cayenne, Cayenne, Guyane française ; 6. Laboratoire hospitalo-universitaire de parasitologiemycologie, Centre hospitalier de Cayenne, Cayenne, Guyane française.

e-mail : alessia.melzani@ch-cayenne.fr

L'histoplasmose est l'infection opportuniste révélatrice de sida la plus répandue en Guyane. Bien que Histoplasma capsulatum ait une distribution mondiale, le syndrome inflammatoire de reconstitution immunitaire associé à l'histoplasmose (IRIS) chez les personnes vivant avec le VIH (pvVIH) semble être rare, même dans les zones d'endémie. L'IRIS est une réaction inflammatoire excessive à un agent pathogène, qui parfois se produit lorsque le système immunitaire est rétabli rapidement après l'instauration d'un traitement antirétroviral. Cette étude visait à décrire les cas d'IRIS associés à l'histoplasmose dans une cohorte de pvVIH résidant en zone d'endémie.

Une étude multicentrique rétrospective a été menée en Guyane du $1^{\mathrm{er}}$ janvier 1997 au 30 septembre 2017. La population source était représentée par les pvVIH inscrits dans la cohorte de pvVIH de Guyane française, qui fait partie de la base de données des hôpitaux français sur le VIH (FHDH). Nous avons inclus les pvVIH adultes ayant présenté un épisode d'histoplasmose dans les six mois suivant le début du traitement antirétroviral. Le critère de jugement principal était la présence ou non d'un IRIS infectieux ou paradoxal selon la définition du rapport du groupe d'experts français. Chaque cas a été décrit à l'aide d'un questionnaire standardisé et soumis à l'accord de deux experts. Tous les patients ont donné leur consentement éclairé. Parmi les 265 cas identifiés et dépistés, 22 cas d'IRIS liés à l'histoplasmose ont été inclus (14 infectieux et 8 paradoxaux) avec un taux d'incidence global de 0,74 pour 1000 personnes-année infectées par le VIH (IC 95 \% : [0,43-1,05]). L'âge moyen était de 40,5 ans. Le sex-ratio H/F était de 1,4. Le délai médian avant la survenue de l'IRIS était de 11 jours [7 à 40] après l'initiation du traitement antirétroviral. Les principales présentations cliniques montraient une maladie disséminée dans un contexte de fièvre sans schéma spécifique. Deux cas graves ont été observés, mais aucun patient n'est décédé dans le mois qui a suivi l'apparition des symptômes d'IRIS. Vingtdeux cas ont été identifiés dans la littérature avec un profil clinique général similaire à celui de notre série. Cependant, les manifestations cutanéomuqueuses étaient plus fréquentes en littérature.

L'incidence de l'IRIS associée à l'histoplasmose était faible, mais a entraîné une morbidité significative chez les pvVIH. Dans les zones d'endémie, le dépistage de l'histoplasmose latente ou subclinique doit être réalisé avant le début du traitement antirétroviral. 


\section{Syndrome hémophagocytaire réactionnel et infection par le VIH en zone tropicale : une série de cas}

Duc Nguyen $^{1}$, A. Buteux ${ }^{1}$, B. Bidaud ${ }^{1}$, M. Pierre-Demar ${ }^{1}$, F. Djossou ${ }^{1}$, P. Couppié ${ }^{2}$, M. Nacher ${ }^{3}$, A. Adenis ${ }^{3}$

1. Service des maladies infectieuses et tropicales, CHAR, Cayenne 97306, Guyane française ; 2. Service de dermatologie, CHAR, Cayenne 97306, Guyane française ; 3. Centre d'investigation clinique, (CIC INSERM 1424), CHAR, Cayenne 97306, Guyane française

e-mail : duc1520@yahoo.fr

Le syndrome hémophagocytaire (SH), appelé également syndrome d'activation macrophagique, secondaire ou réactionnel, est une pathologie rare, mais de mauvais pronostic chez le patient infecté par le VIH. En zone tropicale, cette entité clinicobiologique est peu décrite. L'objectif de cette étude était de décrire les particularités épidémiologiques du SH réactionnel chez les patients infectés par le VIH en région équatoriale sud-américaine.

Une étude observationnelle rétrospective monocentrique a été menée dans le centre hospitalier de Cayenne entre le $1^{\text {er }}$ janvier 2012 et le 30 août 2016. Tout patient adulte et infecté par le VIH ayant un diagnostic de SH réactionnel, et ayant consenti à son inclusion dans la base Dat'Aids (base de données du logiciel Nadis ${ }^{\circledR}$, active depuis 2000), était inclus. Le SH réactionnel était défini par la présence d'au moins trois des huit critères de la classification HLH-2004 et un score diagnostique de SH réactionnel (HScore) supérieur à 169 (sensibilité de $93 \%$ et spécificité de $86 \%$ ).

Quatorze cas de SH réactionnel ont été recensés sur la période d'étude. L'âge moyen était de 46 ans et le sex-ratio de 1,8. L'enquête étiologique des SH réactionnels retrouvait principalement une cause infectieuse (13/14). L'histoplasmose disséminée était retrouvée chez 12 cas sur 14. Le taux de CD4 était inférieur à $250 / \mathrm{mm}^{3}$ chez 13 cas sur 14 . Une charge virale supérieure à 100000 copies/ml était observée dans 13 cas sur 14 . Un traitement précoce par amphotéricine B liposomale était initié dans la majorité des cas $(10 / 14)$. Le taux de létalité à j28 était de $14,2 \%$ : le pronostic global était favorable dans la majorité des cas avec 2 décès parmi les 14 patients.

Pour la première fois, l'histoplasmose est décrite comme la principale étiologie de SH réactionnel chez des patients infectés par le VIH. Si le pronostic du SH réactionnel reste sévère dans cette série de patients infectés par le VIH, la proportion de décès reste très inférieure $(2 / 14,14 \%)$ aux taux de létalité classiquement décrits $(40 \%)$. Ainsi, en zone d'endémie pour l'histoplasmose, un traitement précoce et probabiliste avec de l'amphotéricine B liposomale en première intention pourrait améliorer le pronostic vital de ces patients. Les risques pour la santé liés au séjour en forêt
amazonienne : un sondage en ligne en Guyane

K. Oganov ${ }^{1,2}$, L. Epelboin ${ }^{3}$

1. Service d'accueil des urgences, CHAR, Cayenne 97306, Guyane française ; 2 . Département universitaire de médecine générale, Université des Antilles, Fouillole, Pointe-à-Pitre 97157, Guadeloupe, France ; 3. Service des maladies infectieuses et tropicales, CHAR, Cayenne 97306, Guyane française

e-mail : kirilloganov@gmail.com

La forêt amazonienne a une réputation de lieu de tous les dangers, dont notamment infections tropicales et attaques par la faune sauvage. Les études en milieu hospitalier présentent un biais dû à la sélection des patients les plus sévères. L'objectif de cette étude était d'évaluer les événements de santé directement avec des personnes travaillant en milieu isolé amazonien.

Nous avons mené une enquête en ligne de juillet à septembre 2017 auprès de scientifiques, de naturalistes et d'organismes travaillant en milieu isolé. L'enquête contenait des questions sur les données démographiques, la fréquence et la durée des voyages et les problèmes de santé dans la nature. Les variables continues ont été données en médiane et interquartile.

Quatre-vingt-douze personnes ont répondu à l'enquête, dont $59(64,1 \%)$ étaient des hommes et l'âge médian était de 38,5 ans (28-46). Le temps médian passé en Guyane était de 84 mois $(29,5-192)$. Le biotope le plus fréquenté était la forêt profonde (au moins déplacements occasionnels pour 91,3\% des interrogés), l'activité la plus fréquente était l'étude de la faune et de la flore (au moins activité occasionnelle pour $72,8 \%$ de la population), et la durée du séjour était de 3 et 30 jours pour $68,4 \%$ de la population interrogée.

Les troubles les plus fréquents étaient liés - de manière décroissante - à la faune (plus de 1164 cas signalés, dont plus de 975 cas liés à des insectes), à des pathologies cutanées (plus de 997 cas signalés), à des traumatismes (plus de 268 cas signalés), à maladies infectieuses (plus de 141 cas) et problèmes digestifs (plus de 99 cas). 56,8\% des hospitalisations ont été causées par des problèmes infectieux/parasitaires, suivis par des traumatismes de 22,7 et $9 \%$ par des attaques de la faune. Les problèmes liés aux régions tropicales (maladies non présentes en Europe) représentaient 36,4 \% des admissions, dont 20,5\% étaient liées à la leishmaniose.

Si les événements de santé globaux étaient principalement des attaques de la faune sauvage, des problèmes dermatologiques et des traumatismes ; en revanche, l'infectiologie était la principale cause d'hospitalisation, suivie de la traumatologie. Les voyageurs peuvent être informés de ces risques spécifiques, et une trousse à pharmacie du voyageur doit être adaptée à ces risques. 


\section{Épidémiologie des infections ostéoarticulaires pédiatriques en Guyane française de 2010 à 2015}

L. Osei ${ }^{1}$, N. El Houmami ${ }^{2}$, P. Minodier ${ }^{3}$ A. Sika ${ }^{1}$, T. Basset ${ }^{1}$, H. Seligmann ${ }^{2}$, A. Terraz ${ }^{4}$, M. Demar ${ }^{5}$, J. Pochard ${ }^{6}$, J. Clouzeau ${ }^{7}$, P.-E. Fournier ${ }^{2}$, N. Elenga ${ }^{1}$

1. Service de médecine et chirurgie pédiatrique, CHAR, Cayenne, Guyane française ; 2. Unité de recherche sur les maladies infectieuses et transmissibles émergentes (URMITE), IHU Marseille, France ; 3. Service d'urgences pédiatriques, Hôpital Nord, Marseille, France ; 4. INSERM CIC 1424, CHAR, Cayenne ; 5. Laboratoire de microbiologie, CHAR, Cayenne ; 6. Service de pédiatrie, Centre médico chirurgical de Kourou ; 7. Service de pédiatrie, Centre hospitalier de l'Ouest guyanais

e-mail : lindsay.osei@gmail.com

Nous présentons ici les résultats d'une étude rétrospective effectuée en Guyane française concernant des enfants hospitalisés dans les trois hôpitaux de ce département français d'outre-mer entre janvier 2010 et décembre 2015.

Les dossiers médicaux de 55 enfants ont été analysés : 27 ostéomyélites, 22 arthrites septiques et six infections multifocales et/ou ostéoarthrites. L'âge moyen des patients était de 7,5 ans, avec un ratio garçons/filles de 2,2:1. Quatre-vingts pour cent des enfants inclus de plus de 36 mois ont été admis pour ostéomyélite due à Staphylococcus aureus sensible à la méticilline $(p<0,05)$ secondaire à une infection cutanée négligée. Cinq enfants ont présenté des infections multisystémiques (dont un décès) dues à des souches de $S$. aureus productrices de leucocidine de Panton-Valentine $(p<0,01)$. En revanche, les enfants âgés de 6 à 36 mois présentaient plus d'infections sans germe pathogène identifié $(p<0,05)$, ainsi qu'un taux plus important d'arthrites septiques, avec des signes cliniques et biologiques peu sévères, ce qui serait compatible avec des infections à Kingella kingae.

Des études prospectives sont nécessaires afin de mieux guider les stratégies diagnostiques et thérapeutiques.

\section{Conséquences de l'infection à virus Zika sur la grossesse : états des lieux en Guyane française au premier semestre 2016}

\section{J. Pasquier ${ }^{1,2}$, H. Hilderal $^{1}$, A. Fahrasmane ${ }^{1}$, S. Dabrowski ${ }^{1}$,} D. Rousset ${ }^{3}$, F. Djossou ${ }^{4}$, M. Nacher ${ }^{1}$, M. Douine ${ }^{1}$

1. Unité INSERM, CIC 1424, CHAR, Cayenne 97306, Guyane française ; 2 . Département universitaire de médecine générale, Université des Antilles, Fouillole, Pointe-à-Pitre 97157, Guadeloupe, France ; 3. Laboratoire de virologie, Institut Pasteur de la Guyane, Cayenne, Guyane française ;
4. Service des maladies infectieuses et tropicales, CHAR, Cayenne 97306, Guyane française

e-mail : jeremiepasquier@gmail.com

Une épidémie de virus Zika (ZIKV) a eu lieu en Amérique du Sud en 2015 et 2016. Des complications fotales à tropisme essentiellement neurologiques peuvent apparaître en cas d'infection maternelle à ZIKV durant la grossesse. Depuis janvier 2016, l'épidémie sévissait en Guyane française.

Une étude prospective, descriptive, multicentrique, ZIKA-DFA-FE a été mise en place pour étudier les conséquences d'une infection maternelle à ZIKV durant la grossesse. Ce travail présente une analyse partielle des données de cette étude, entre le 4 avril et le 30 juin 2016, en Guyane française.

Cent neuf patientes ayant eu une infection à Zika au cours de la grossesse, diagnostiquée par RT-PCR ou sérologie, ont été incluses. Quarante-deux patientes $(38,5 \%)$ ont été symptomatiques. Soixante-neuf patientes $(63,3 \%)$ ont accouché d'un enfant neurologiquement normal à la naissance, 14 $(12,8 \%)$ ont subi des pertes de grossesses. Quatorze patientes $(12,8 \%)$ ont présenté des anomalies échographiques, deux se sont avérées être des anomalies sévères du développement, dont une microcéphalie.

Cette analyse préliminaire montre une tendance à des pertes fotales et à des anomalies du développement fotal en cas d'infection maternelle à ZIKV. Il conviendra d'attendre la fin de l'étude ZIKA-DFA-FE pour obtenir des effectifs plus importants.

\section{La question du diagnostic en situation transculturelle : à propos d'une observation}

C. Payen ${ }^{1}$, P. Patillot ${ }^{1}$, R. Radjack ${ }^{1,2}$

1. Service de psychiatrie, CHAR, Cayenne 97306, Guyane française ; 2. Service de pédopsychiatrie transculturelle, Maison de Solenn, la maison des adolescents de Cochin (APHP), 97 bld de Port-Royal, 75014 Paris, France

e-mail : claire.payen@live.fr

Nous étudions l'observation de Mme S., 28 ans, haïtienne, hospitalisée sous contrainte dans le service de psychiatrie fermé de l'hôpital de Cayenne (Guyane) pour trouble psychotique bref. Présentant des céphalées, une fluctuation thymique, un vécu persécutif, des idées mystiques envahissantes (punition divine), associées à des hallucinations auditives et visuelles, la famille demande son hospitalisation, parallèlement à l'administration de remèdes traditionnels. Cela survenait dans un contexte de post-partum. Après apaisement des symptômes, elle sort sous décanoate d'halopéridol, puis est perdue de vue. 
Ce cas clinique illustre à première vue un trouble psychotique bref (DSM-5). L'abondance des éléments culturels soulève la question de leur impact dans l'évaluation diagnostique. Différentes hypothèses sont évoquées grâce à une analyse complémentariste (Devereux) mettant en regard les étiologies traditionnelles avec les diagnostics occidentaux : troubles psychotique partagé, bipolaire, dépressif, du postpartum, état de stress post-traumatique, personnalité histrionique, système de croyances ou de syndromes liés à la culture (le move sanou " énervement" en créole haïtien). Retenons que le discours religieux respectait la logique culturelle d'appartenance, et que les éléments thymiques présentaient une variabilité symptomatique (idées de persécution et de culpabilité associées à des symptômes somatiques). Un trouble dépressif, récurrent (antécédent similaire quatre années auparavant), du post-partum, avec codage culturel, semblait donc plus approprié. Seules l'évolution et la prise en compte des éléments culturels de manière simultanée lors de l'hospitalisation auraient permis d'affiner le diagnostic.

Point particulier : la distance culturelle entre le patient et le clinicien influe, elle aussi, sur l'élaboration d'un diagnostic, en lien avec les préjugés et stéréotypes soutenus par leurs identités individuelle, sociale et culturelle, ainsi que par l'histoire de leur pays (immigration mal perçue). Ces facteurs peuvent être à l'origine de pseudodiagnostics marqués par le racisme institutionnel, comme « l'hystérie du migrant haïtien » dont Mme S. était affublée. À nous d'analyser nos contre-transferts culturels, de pratiquer le décentrage, voire de nous aider de l'entretien de formulation culturelle afin d'éviter toute situation d'errance diagnostique.

\section{Expositions sexuelles aux risques viraux prises en charge au centre hospitalier de Cayenne : étude rétrospective 2015-2016}

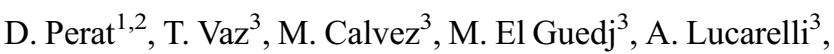 \\ M. Nacher ${ }^{1,4}, H$. Florence ${ }^{1,5}$
}

1. COREVIH Guyane, CHAR, Guyane française ; 2. Département universitaire de médecine générale, Faculté de médecine Hyacinthe Bastaraud, Université des Antilles, Fouillole, Pointe-à-Pitre 97157, Guadeloupe, France, Antilles françaises ; 3. Hôpital de jour, CHAR, Cayenne, Guyane française ; 4. Centre d'investigation clinique épidémiologie clinique Antilles Guyane, INSERM CIC 1424, CHAR, Guyane française ; 5. Reseau Kikiwi, Cayenne, Guyane

e-mail : david.perat@zaclys.net

La Guyane est le département français le plus affecté par le VIH et les violences sexuelles. Les recommandations concernant les accidents d'exposition aux risques viraux (AEV) de type sexuel conduisent à un large recours au trai- tement postexposition (TPE). L'objectif de cette étude était de faire un état des lieux de la prise en charge des AEV sexuels à Cayenne. Il s'agit d'une étude monocentrique rétrospective. Tout patient ayant consulté au centre hospitalier $(\mathrm{CH})$ de Cayenne dans les 72 heures suivant un AEV sexuel entre le $1^{\text {er }}$ janvier 2015 et le 31 décembre 2016 a été inclus.

En 2015-2016, 323 patients ont consulté pour AEV sexuel au $\mathrm{CH}$ de Cayenne (3,10 par semaine en moyenne), augmentation de $11,11 \%$ entre 2015 et 2016 . Sex-ratio : 0,76 ; âge médian : 26 ans ; mineurs : $16,4 \%$; nés à l'étranger : 43,0\%. Couverture maladie : aucune (hommes/femmes) : 17,0/28,8\% ; aide médicale d'État (hommes/femmes) : $0,7 / 7,1 \%(p<0,001)$. Délai médian expositionconsultation : 12 heures ; patient source VIH positif ou suspecté tel : $6,2 \%$; source présente à la consultation $(2,8 \%)$; rapports hétérosexuels $(93,8 \%)$, anaux $(20 \%)$, oraux $(6,5 \%)$; non-usage du préservatif : $38,4 \%$; relation transactionnelle : $15,0 \%$; agression sexuelle : 30,0 \% (femmes : $49,5 \%$; mineurs : 88,8 \%). TPE VIH initié : $86,107 \%$; trithérapie comportant un inhibiteur de protéase : $70,107 \%$; contraception postcoïtale : $44,5 \%$; vaccin VHB : une dose $(5,9 \%)$, deux doses $(1,24 \%)$, immunoglobulines anti-VHB (0\%). Suivi complet : $6,2 \%$. Aucune séroconversion documentée.

Nombreux recours pour AEV sexuels au $\mathrm{CH}$ de Cayenne en 2015-2016 (incidence estimée : 12,24/100 004 habitants par année). Agressions sexuelles et relations transactionnelles fréquentes. Population singulière : jeune, majoritairement féminine et quasi exclusivement hétérosexuelle. Inégalités d'accès aux soins marquées entre hommes et femmes. Large initiation de TPE anti-VIH. Faible rétention des patients. Prises en charge centrées sur le VIH, risque de grossesse et d'infection par le VHB rarement pris en compte.

\section{État des lieux de la prise en charge de la mort inattendue du nourrisson en Guyane, de 2006 à 2016}

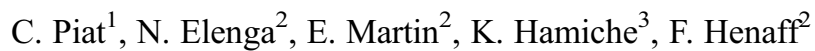

1. Service d'accueil des urgences, CHAR, Cayenne 97306, Guyane française ; 2. Service de pédiatrie, CHAR, Cayenne 97306, Guyane française ; 3. Unité médico-judiciaire, CHAR, Cayenne 97306, Guyane française

e-mail : campt@hotmail.fr

La mort inattendue du nourrisson (MIN) est un problème de santé publique pour lequel les recommandations françaises de prise en charge datant de 2007 sont difficilement applicables en Guyane. L'objectif principal de notre étude était de décrire les modalités de prise en charge médicale et 
médicolégale en cas de MIN en Guyane et de proposer un protocole adapté aux particularités départementales, tant géographiques qu'épidémiologiques.

Nous avons réalisé une étude rétrospective, observationnelle et multicentrique de tous les dossiers de MIN sur l'ensemble des établissements de soins du département (hôpitaux et centres de santé), entre le $1^{\text {er }}$ janvier 2006 et le 31 décembre 2016. Toutes les données des dossiers médicaux préhospitaliers, hospitaliers et médicolégaux ont été recueillies.

Quarante-quatre dossiers ont été analysés. L'âge médian était de 130 jours, soit 4,3 mois et le sex-ratio H/F de 1,58. Soixante-cinq pour cent des décès ont eu lieu à domicile. Trente-trois nourrissons étaient domiciliés dans les communes du littoral avec un centre hospitalier à proximité et 11 nourrissons venaient de communes isolées. Quinze nourrissons ( $34 \%$ ) ont eu une prise en charge préhospitalière par le service mobile d'urgence et de réanimation (Smur). Des manœuvres de réanimation ont été entreprises pour 26 nourrissons, soit (59\%). Quarante-trois pour cent des nourrissons ont eu un bilan biologique standard, la ponction lombaire a été réalisée dans $38 \%$ des cas, et $36 \%$ des nourrissons ont eu un scanner corps entier. Aucune imagerie cérébrale n'a été réalisée de manière isolée. Une autopsie médicolégale a été réalisée chez 14 nourrissons, soit $31 \%$. Aucune autopsie scientifique n'a été réalisée. Seulement quatre familles ont été vues en entretien afin de leur transmettre les résultats des examens effectués et éventuellement la cause du décès si elle avait été retrouvée.

L'absence de centre de référence pour les MIN en Guyane est un frein à une prise en charge adéquate de ces situations. La prise en charge dépend ainsi généralement uniquement du médecin présent sur les lieux et de ses connaissances sur le sujet. Trop peu d'examens sont réalisés, et ce d'autant plus dans les communes isolées, en partie par manque de moyens techniques et de la difficulté d'accessibilité des communes (pirogue, avion, hélicoptère). De même, aucune autopsie scientifique n'est réalisée par manque de médecins formés dans ce domaine. Il semble dès lors indispensable d'élaborer un protocole de prise en charge multidisciplinaire, au plus proche des recommandations professionnelles, mais adapté à l'offre de soins guyanaise.

\section{Épidémiologie et pronostic des encéphalites en Guyane française}

A. Roux ${ }^{1,2}$, S. Houcke $^{3}$, A. Sanna ${ }^{4}$, C. Mathien ${ }^{3}$, C. Mayence, ${ }^{3}$, R. Gueneau ${ }^{3}$, G. Liegeon ${ }^{3}$, G. Walter ${ }^{5}$, D. Resiere $^{6}$, N. Elenga ${ }^{7}$, G. Resin ${ }^{7}$, F. Djossou ${ }^{5}$, D. Hommel ${ }^{3}, \mathrm{H}$. Kallel ${ }^{3}$

1. Service d'accueil des urgences, CHAR, Cayenne, Guyane française ; 2. Département universitaire de médecine géné- rale, Université des Antilles, Fouillole, Pointe-à-Pitre 97157, Guadeloupe, France ; 3. Réanimation polyvalente adulte, CHAR, Cayenne ; 4. Agence régionale de santé, Cayenne, Guyane française ; 5 . Unité des maladies infectieuses et tropicales, CHAR, Cayenne ; 6. Réanimation polyvalente adulte, CHU Martinique ; 7. Pédiatrie, néonatalogie, CHAR, Cayenne

\section{e-mail : alexandre.roux@ch-cayenne.fr}

L'encéphalite est un enjeu important de santé publique, mais son diagnostic est difficile, avec une présentation clinique hétérogène, et un grand nombre d'étiologies possibles. Ces étiologies dépendent d'une variabilité régionale, particulièrement pour les maladies transmissibles et les zoonoses. Le but de notre étude était de décrire l'épidémiologie régionale et le pronostic des encéphalites en Guyane française.

Notre étude était rétrospective, descriptive et portait sur la population générale de Guyane française, les patients hospitalisés à l'hôpital général au centre hospitalier de Cayenne, de janvier 2007 à juillet 2017. Les patients étaient inclus selon la définition du consortium international de 2013 et le pronostic était évalué à trois mois par le Glasgow Outcome Scale.

Ont été inclus 108 patients, soit une incidence approximative de 4 cas/100 000 habitants par année. L'âge moyen était de 38,3 $\pm 20,5$ ans ( $\min : 7$ mois ; $\max : 80$ ans). Dix-huit patients $(16,7 \%)$ avaient moins de 16 ans. Vingt-cinq patients $(23,1 \%)$ venaient des communes de l'intérieur (isolement géographique). Soixante-huit patients (63\%) avaient au moins un antécédent, et $41,7 \%$ vivaient avec le VIH. L'étiologie des encéphalites était identifiée dans 81 cas (75\%), avec 72 causes infectieuses (66,7\%). Les agents infectieux les plus communément identifiés étaient : Cryptococcus $(18,5 \%)$ - quel que soit le statut immunitaire -, Toxoplasma gondii $(13,9 \%)$, Streptococcus pneumoniae (5,5\%), VZV et HIV (3,7\% chacun), HSV et Mycobaterium tuberculosis $(2,8 \%$ chacun). À trois mois, 48 patients $(46,6 \%)$ ont eu une évolution défavorable. Les facteurs de risque indépendamment associés à l'évolution défavorable étaient de vivre dans les communes de l'intérieur $(p=0,036 ; \mathrm{OR}=4,19 ; \mathrm{IC} 95 \%=[1,09-16,06])$, le recours à la ventilation mécanique $(p=0,002 ; \mathrm{OR}=5,92 ; \mathrm{IC} 95 \%$ $=[1,95-17,95])$, et l'âge $\geq 65$ ans $(p=0,049 ; \mathrm{OR}=3,99$; IC $95 \%=[1,01-15,9])$.

L'épidémiologie des encéphalites en Guyane française est différente de celle qui est décrite ailleurs dans le monde : la cause la plus fréquente était liée à la cryptococcose. Il est primordial de poursuivre la surveillance d'agents émergents, en particulier pour les populations de l'intérieur. La rédaction de guides de prise en charge des encéphalites en Guyane française doit être discutée. 
Envenimation par une chenille Lonomia sp. en Guyane française d'évolution favorable après usage d'un sérum antivenin brésilien : un cas réussi de coopération internationale pour un risque tropical rare, mais mortel

C. Mayence ${ }^{1}$, C. Mathien ${ }^{1}$, A. Sanna ${ }^{2}$, S. Houcke ${ }^{1}$, P. Tabard ${ }^{2}$, A. Roux ${ }^{1}$, C. Valentin ${ }^{3}$, D. Resiere ${ }^{4}$, D. Lemonnier $^{5}$, F. Nkont $\mathrm{Cho}^{5}$, D. Hommel ${ }^{1}$, H.W. Fan ${ }^{6}$, H. Kallel ${ }^{1}$

1. Intensive Care Unit, Cayenne General Hospital ; 2. Regional Authority of Health, French Guiana ; 3. Emergency Department, Kourou Hospital ; 4Intensive Care Unit, Fort de France University Hospital, Martinique ; 5. Pharmacy Department, Cayenne General Hospital, French Guiana ; 6. Butantan Institute, Sao Paulo, Brazil
Sur le continent américain, les formes larvaires (chenilles) du genre Lonomia peuvent provoquer des réactions systémiques chez l'Homme.

Nous rapportons le troisième cas d'envenimation par Lonomia sp. enregistré en Guyane française en 25 ans et le premier dans lequel un antivenin spécifique importé du Brésil a été administré. Des symptômes graves d'envenimation ont été observés chez ce patient, notamment douleur, coagulopathie et hémorragie systémique. Ils sont causés par le contact cutané avec les chenilles. L'évolution a toutefois été rendue possible grâce à la coopération internationale des autorités sanitaires françaises et brésiliennes. 